\title{
Nachruf auf Gaetano Benedetti
}

\section{Michael Ermann}

Online publiziert: 4. Februar 2014

(c) Springer-Verlag Berlin Heidelberg 2014

\begin{abstract}
„Auf diese existentielle Frage, die jeder psychisch kranke Patient an mich stellt unbewusst, aber von mir als solche erlebt - kann ich als Therapeut und Mitmensch nur mit dem Einsatz meiner gesamtpersönlichen Existenz antworten..." Das schreibt ein Mann, der mit seinem persönlichen Einsatz für seine Patienten tiefe Eindrücke hinterlassen hat: Gaetano Benedetti. Wer ihm in einem seiner Vorträge zugehört hat oder wer ihm persönlich begegnet ist, wird diese Erfahrung niemals vergessen: Die kleine, im Alter hagere Gestalt, das kantige Auftreten, seine harte, laute, durch den italienischen Akzent geprägte Sprache mit dem tiefen rollenden Rrr - und ganz im Gegensatz dazu seine filigran zu nennenden Gedanken, in denen er seine Botschaft herüberbrachte in einer ungeahnten Sensibilität für die düstere Welt der Psychose und für Träume. All das ist unvergessen und wird sein Leben überdauern.

Im Jahre $1920 \mathrm{im}$ sizilianischen Catania geboren, arbeitete Gaetano Benedetti nach seinem Studium mehr als 60 Jahre lang als Psychiater, Psychotherapeut und Psychoanalytiker. Als Schüler von Manfred Bleuler am Burghölzli in Zürich wandte er sich der Psychopathologie und der Psychodynamik zu und absolvierte bei Gustav Bally seine Lehranalyse, wodurch er assoziiertes Mitglied der IPA wurde. Nach zweifacher Habilitation in Zürich und Rom erhielt er 1956 den Ruf an die Universität Basel. Er hatte die Leitung der Abteilung für Psychohygiene und Psychotherapie bis zu seiner Emeritierung im Jahre 1985 inne und gab ihr ein psychoanalytisches Gepräge.

Die Ausbildung von jungen Analytikern in Mailand ab den 1960er Jahren führte 1971 dort zur Gründung eines Instituts für Psychoanalytische Studien, an dem auch Johannes Cremerius tätig wurde. Sie stellte einen Markstein in der Entwicklung der Psychoanalyse in Italien dar. Hier wurden Generationen von Psychoanalytikern aus-
\end{abstract}

Prof. Dr. med. M. Ermann ( $\bowtie)$

München, Deutschland

E-Mail: Ermann-Forum@t-online.de 
gebildet, die durch den mutigen, kreativen und flexiblen Stil von Benedetti zutiefst geprägt wurden und die sich besonders der Behandlung schwerer Persönlichkeitsstörungen widmeten.

Benedettis Schwerpunkt war die psychoanalytisch begründete Behandlung von Psychosen und die Arbeit mit Träumen. Hier verband er psychoanalytische Ansätze mit der Phänomenologie und Imagination. Davon zeugen seine Monografien - ich nenne nur eine: Todeslandschaften der Seele (1983), die uns als junge Analytiker zutiefst beeindruckte - und hunderte von Vorträgen und Publikationen. Sein Lebenswerk hat der psychoanalytischen Traumlehre und der Psychosentherapie neue und innovative Impulse gegeben.

Neben anderen Auszeichnungen wurde er 1983 Ehrenmitglied der Deutschen Psychoanalytischen Gesellschaft DPG und Fellow der American Academy of Psychoanalysis. Seit der Gründung des Forum der Psychoanalyse 1984 gehörte er ihrem Beirat an. Wie hoch sein Ansehen in der Fachwelt war, belegt eine Initiative Münchener Psychoanalytiker aus dieser Zeit, die ihn für den Nobelpreis vorschlagen wollten.

Viele Jahre lang war er in Begleitung seiner Frau auch noch im Ruhestand zu Vorträgen gereist, mit denen er in einer psychiatrischen Fachwelt zunehmender Rationalität seine Botschaft von der Psychotherapie als existenzieller Herausforderung unbeirrt vertrat, bis in den letzten Jahren seine körperlichen und geistigen Kräfte nachließen. Benedetti starb am 2. Dezember in Basel. Sein Tod hinterlässt in der psychoanalytischen Welt eine Lücke, die nicht zu schließen sein wird. Wer ihn kannte, wird ihn vermissen. 